ARTIGO ORIGINAL

\title{
PERFIL EPIDEMIOLÓGICO DA LEISHMANIOSE VISCERAL NO BRASIL NO PERÍODO DE 2007 A 2017 \\ EPIDEMIOLOGICAL PROFILE OF VISCERAL LEISHMANIASIS IN BRAZIL IN THE PERIOD FROM 2007 TO 2017
}

NOELY DE CARVALHO LEITE', JORDANA LUCIO GARCIA', ITAMAR MAGALHÃES GONÇALVES ${ }^{2}$

\section{RESUMO}

Introdução: A Leishmaniose Visceral (LV) é uma antropozoonose generalizada, de evolução crônica e sistêmica, que, apesar de ser passível de tratamento e cura, possui elevadas taxas de incidência e letalidade. Tipicamente, afeta países subdesenvolvidos, com uma preferência pela população mais vulnerável socioeconomicamente, e possui ampla distribuição territorial, sendo o Brasil um dos principais países acometidos. O objetivo do artigo é analisar dados epidemiológicos da doença no país no período estabelecido. Metodologia: Trata-se de um estudo epidemiológico descritivo, realizado a partir de busca de dados de acesso livre do Sistema de Informação de Agravos de Notificação no período de janeiro de 2007 a dezembro de 2017. Resultados: No intervalo em questão, foram documentados 40.786 casos de LV, com uma variação pouco heterogênea entre os anos estudados. Destes, 2011 registrou o maior número de casos, contando com 4.039 registros. As populações mais afetadas incluem: sexo masculino (média de 2.362 casos/ano), faixa etária de 1-4 anos (média de 978 casos/ano), raça parda (média de 2.750 casos/ano) e baixa escolaridade - a maioria entre a primeira e a quarta série (média de 405 casos/ano). Além disso, excluindo-se os casos ignorados/brancos, $10 \%$ do total dos indivíduos apresentaram coinfecção da LV com HIV. A região Nordeste demonstrou maior incidência da doença, com $52,7 \%$ do total dos casos. O principal estado atingido é o Maranhão, que apresentou um número de 6.070 casos durante o período estudado. Discussão: Considerando a relevância do alto número de casos apontados nesse trabalho, mostra-se necessária a implantação de políticas públicas voltadas para a prevenção da transmissão, busca ativa de casos e educação em saúde. Conclusões: Nesse quadro, para maior eficácia, deve existir integração entre vigilância epidemiológica e assistência clínica, acesso aos serviços, capacitação dos profissionais e empoderamento da população.

Palavras-chave: Leishmaniose visceral; Epidemiologia; Doenças Negligenciadas; Zoonose.

${ }^{2}$ Graduado em Medicina pela Universidade Federal de Uberlândia (2000) e Especialização em Cirurgia Geral e Urologia. Professor Titular do Curso de Medicina da ITPAC Palmas e Perito Médico-Legista pela Secretaria de Segurança Pública do Estado do Tocantins. Mestre em Ensino em Ciências da Saúde pela Universidade Federal de São Paulo. Instituto Tocantinense Presidente Antônio Carlos - ITPAC Palmas, Palmas, Tocantins, Brasil.

Autor Correspondente: Noely de Carvalho Leite, noelydecarvalho@hotmail.com

Editor: Carvalho A. A. B. Medicina, Universidade Federal do Tocantins, Brasil.

Publicado: 23 de fevereiro de 2021.

Direitos Autorais: (C) 2021 Leite et al. Este é um artigo de acesso aberto que permite o uso, a distribuição e a reprodução sem restrições em qualquer meio, desde que o autor original e a fonte sejam creditados.

Conflito de interesses: os autores declararam que não existem conflitos de interesses.

\begin{abstract}
Introduction: Visceral Leishmaniasis (VL) is a generalized anthropozoonosis, of chronic and systemic evolution, which, despite being amenable to treatment and cure, has high rates of incidence and lethality. Typically, it affects underdeveloped countries, with a preference for the most socioeconomically vulnerable population, and has a wide territorial distribution, with Brazil being one of the main countries affected. The objective of the article is to analyze epidemiological data on the disease in the country in the period established. Methodology: This is a descriptive epidemiological study, carried out based on the search for free access data from the Notifiable Diseases Information System from January 2007 to December 2017. Results: In the interval in question, 40,786 were documented cases of VL, with a slightly heterogeneous variation between the years studied. Of these, 2011 recorded the largest number of cases, with 4,039 records. The most affected populations include: male gender (average of 2,362 cases / year), age range of 1-4 years (average of 978 cases / year), brown race (average of 2,750 cases / year) and low education - most among the first and the fourth series (average of 405 cases / year). In addition, excluding the ignored / white cases, $10 \%$ of the total individuals had co-infection of the VL with HIV. The Northeast region showed a higher incidence of the disease, with $52.7 \%$ of the total cases. The main state affected is Maranhão, which presented a number of 6,070 cases during the period studied. Discussion: Considering the relevance of the high number of cases mentioned in this work, it is necessary to implement public policies aimed at preventing transmission, active search for cases and health education. Conclusions: In this context, for greater effectiveness, there must be integration between epidemiological surveillance and clinical assistance, access to services, training of professionals and empowerment of the population.
\end{abstract}

Keywords: Visceral leishmaniasis; Epidemiology; Neglected diseases; Zoonoses 
A Leishmaniose Visceral (LV) é uma antropozoonose generalizada, de evolução crônica e sistêmica ${ }^{1}$ que, em razão de sua heterogeneidade epidemiológica, ampla e distinta distribuição territorial, elevadas taxas de incidência e letalidade $^{2}$, tem se tornado uma das mais importantes doenças da atualidade. No Brasil, está presente em todas as regiões, alcançando cerca de 21 Unidades Federativas. Em 2017, os dados gerados dos últimos dez anos indicavam a ocorrência dos surtos ocorridos nas cidades do Rio de Janeiro $(\mathrm{RJ})$, Belo Horizonte (MG), Araçatuba (SP), Santarém (PA), Corumbá (MS), Teresina (PI), Natal (RN), São Luís (MA), Fortaleza (CE), Camaçari (BA) e as epidemias ocorridas nos municípios de Três Lagoas (MS), Campo Grande (MS) e Palmas $(\mathrm{TO})^{3}$.

Os agentes etiológicos da leishmaniose visceral são protozoários tripanosomatídeos do gênero Leishmania ${ }^{4}$ e a transmissão ao homem e ao cão - principal reservatório do parasita - ocorre por meio da picada dos vetores Lutzomyia cruzi e, principalmente, Lutzomyia longipalpis, inseto flebotomíneo hematófago encontrado nas cinco regiões do Brasil ${ }^{1}$.

Além disso, a LV é típica de regiões tropicais e subtropicais, e afeta principalmente países subdesenvolvidos ${ }^{1}$, onde as transformações no ambiente, provocadas pelo intenso processo de urbanização, por pressões socioeconômicas, pobreza consequente de distorções na distribuição de renda, condições de saneamento precárias, esvaziamento rural e as secas periódicas acarretam a expansão das áreas endêmicas e a facilidade de propagação, favorecendo a ocorrência de epidemias. Tal qual no Brasil, a expansão do processo de urbanização - principalmente das zonas periféricas e transicionais dos grandes centros - em meados da década de 1970, propiciou o advento de transformações ambientais antropogênicas e de acelerado êxodo rural para os subúrbios urbanos, o que acarretou o deslocamento e a adequação tanto do vetor quanto dos reservatórios do protozoário ${ }^{5}$.

Nesse contexto, a relevância da intensificação de estudos sobre a LV no Brasil está em crescente expansão pela sua facilidade de provocar epidemias ${ }^{4}$ e atingir principalmente a população mais pobre ${ }^{6}$. Além disso, o Ministério da Saúde inclui essa doença como uma das prioridades de pesquisa e atuação em doenças negligenciadas, confirmando a necessidade do desenvolvimento de estratégias de controle mais específicas e eficazes ${ }^{7}$.

Considerando a alta incidência e dificuldades de controle, novas propostas de vigilância e medidas de prevenção devem ser estabelecidas a partir de uma delimitação mais específica, que considere as determinadas condições de vulnerabilidade envolvidas no contágio da LV e o enfoque epidemiológico das áreas do país, sejam elas de transmissão ou de risco, para que políticas públicas singulares sejam efetivadas em suas respectivas localizações.

\section{OBJETIVOS}

O objetivo do artigo é analisar dados epidemiológicos da doença no país no período estabelecido
Trata-se de um estudo epidemiológico descritivo, que procura indicar a distribuição da LV, investigando a prevalência da mesma segundo determinadas características dos indivíduos acometidos. A pesquisa valeu-se de dados do Brasil. O país está localizado na América do Sul e ocupa uma área territorial de $8.510 .820,623 \mathrm{~km}^{2}$, com uma população estimada de 210.147 .125 pessoas $^{8}$.

Realizou-se a busca de dados de acesso livre do Sistema de Informação de Agravos de Notificação (SINAN), encontrado no Departamento de Informática do Sistema Único de Saúde (DATASUS), para a obtenção do número de casos confirmados de LV no Brasil de janeiro de 2007 a dezembro de 2017. Durante essa investigação, foram analisadas variáveis fornecidas pelo próprio SINAN - incluindo sexo, raça, faixa etária, nível de escolaridade, coinfecção com HIV, tipo de entrada, evolução, diagnóstico, critério de confirmação e distribuição geográfica por UF e por região de saúde. Foram incluídos os casos confirmados e notificados de LV por meio do SINAN, ocorridos nos anos de 2007 a 2017.

A seguir, esses dados foram mapeados com base em planilhas eletrônicas oferecidas pelo TabWin, programa disponibilizado pelo próprio DATASUS que efetua tabulações das informações e proporciona o levantamento de especificidades epidemiológicas, para a realização da análise estatística descritiva do estudo pelo Microsoft Excel. Após tabulação, converteu-se essas informações em gráficos e os resultados foram finalmente interpretados e analisados para a compreensão do perfil epidemiológico da doença.

\section{RESULTADOS}

Durante o período analisado, de 2007 a 2017, foram documentados um total de 40.786 casos de LV no Brasil, sendo a variação entre os anos pouco heterogênea. Destes, o ano de 2011 registrou o maior número de casos, contando com 4.039 registros. A maior proporção se deu no sexo masculino, apontando uma média de aproximadamente 2.362 casos por ano, enquanto no sexo feminino houveram 1.346 casos registrados em média por ano (gráfico 1).

Gráfico 1- Número de casos de LV de acordo com o primeiro ano dos sintomas, de 2007 a 2017, conforme o sexo.

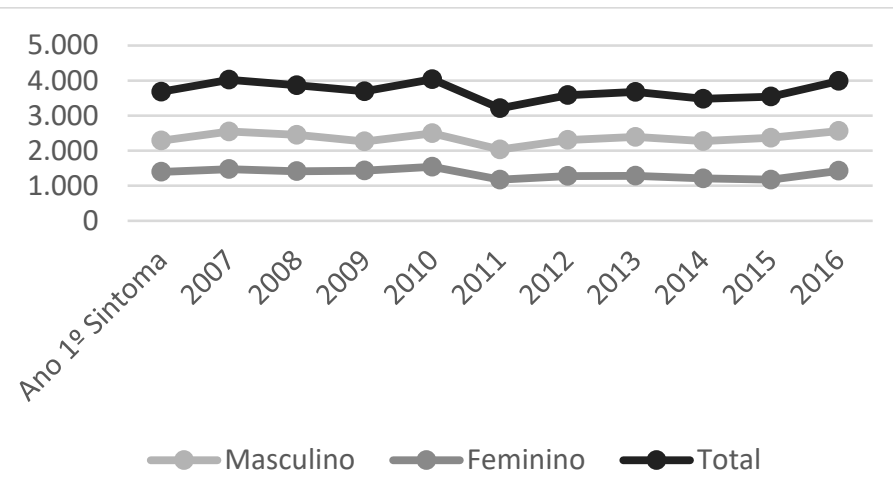

Fonte: Ministério da Saúde, Sistema de Informação de agravos de Notificação - SINAN, 2020 
Quanto à faixa etária (tabela 1), as idades de 1-4 anos e de 20-39 anos foram as mais acometidas, com $48 \%$ do total. A primeira com um total de 10.756 ocorrências confirmadas durante o período em questão, correspondendo a $26 \%$ do total, e uma média de cerca de 978 casos/ano. Já nas idades de 20-39 anos, foram documentados 9.004 eventos e uma média de 818 casos/ano. As demais faixas etárias possuem $51 \%$ do total, variando de 6.502 casos nos pacientes de $40-59$ anos a 387 casos nos pacientes com 80 anos ou mais.

Tabela 1- Número e porcentagem de casos de LV registrados nos anos de 2007 a 2017 no Brasil de acordo com a faixa etária do paciente

\begin{tabular}{lcc}
\hline Faixa etária & $\mathbf{N}^{*} \mathbf{( 4 0 7 8 5 )}$ & $\%$ \\
\hline$<1$ ano & 3695 & 9,05 \\
$1-4$ anos & 10756 & 26,37 \\
$5-9$ anos & 3936 & 9,65 \\
$10-14$ anos & 1983 & 4,86 \\
$15-19$ anos & 1989 & 4,87 \\
$20-39$ anos & 9004 & 22,07 \\
$40-59$ anos & 6502 & 15,94 \\
$60-64$ anos & 918 & 2,25 \\
$65-69$ anos & 719 & 1,76 \\
$70-79$ anos & 876 & 2,14 \\
80 e + & 387 & 0,94 \\
\hline
\end{tabular}

Fonte: Ministério da Saúde, Sistema de Informação de agravos de Notificação - SINAN, 2020

Em relação à raça, a população parda deteve 27.503 registros entre 2007 e 2017 , o que corresponde a $67,4 \%$ do total de casos. Na sequência, brancos manifestaram 6.056 casos, pretos 3.275 casos, indígenas 397 casos e amarelos 303 casos, além dos 3.252 casos cuja raça foi ignorada. Quanto ao nível de escolaridade dos pacientes, a maioria deles estava entre a primeira e a quarta série, totalizando 4.058 casos, o que corresponde a cerca de $26 \%$ dos casos - com exceção dos registros em que essa variável não se aplica ou é ignorada, que totalizam 25.255 casos.

Além disso, 3.030 indivíduos apresentaram coinfecção da LV com HIV, sendo estes $10 \%$ do total, excluindo-se os casos ignorados/brancos. Dessa variável, 10.660 dos casos foram pontuados como ignorados/brancos. Quando analisado o tipo de entrada, 36.976 corresponde a casos novos, cerca $90 \%$ dos 40.786 registrados em todos os anos.

Analisando-se os critérios de confirmação, dos 40786 confirmados, 35197 casos que correspondem a cerca de $86 \%$, tiveram critério de confirmação laboratorial, enquanto os demais 5578 casos contaram com critério clínicoepidemiológico como confirmação de diagnóstico.

Em relação ao estado de residência, o estado do Maranhão conta com o maior número de casos na totalidade dos anos, com 6070, seguido de Minas Gerais com 5719 e do Ceará com 5672. O Maranhão carrega consigo cerca de $15 \%$ do total de registro, esses três estados com maior incidência possuem um total de $38 \%$ dos registros. A região Nordeste, com 9 estados, possui um total de 21.496 casos, o que corresponde a $52,7 \%$ do total (gráfico 2) e conta com dois dos primeiros três estados de maior incidência.

Quanto aos demais estados, Amapá foi o que obteve o menor número, com 4 casos, seguido de Santa Catarina com
12, Rondônia e Amazonas ambos com 13 registros. Em escala decrescente, Pará (3753), Bahia (3716), Tocantins (3632), Piauí (2409), São Paulo (2340), Mato Grosso do Sul (2289), Pernambuco (1173), Rio Grande do Norte (998), Sergipe (655), Goiás (488), Mato Grosso (441), Paraíba (425), Alagoas (378), Roraima (185), Distrito Federal (169), Espírito Santo (71), Rio de Janeiro (67), Paraná (40), Rio Grande do Sul (30).

Gráfico 2- Número de casos registrados no Brasil na região de residência de acordo com o ano do primeiro sintoma no decorrer dos anos 2007 a 2017

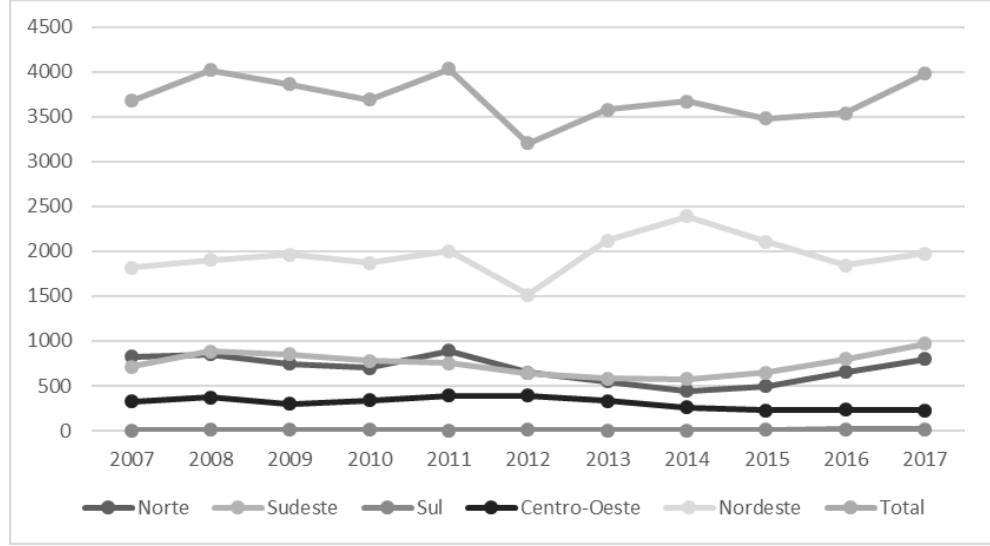

Fonte: Ministério da Saúde, Sistema de Informação de agravos de Notificação - SINAN, 2020

No período analisado, as regiões de saúde com maior número de casos foram a região "1a Região Fortaleza" no Ceará (1999 casos), a região "Campo Grande" no Grosso do Sul (1882 casos) e a região "Médio Norte Araguaia" no Tocantins (1675 casos). Percebe-se também, de maneira geral, uma discrepância de registros entre diferentes regiões do mesmo estado. O Ceará, por exemplo, que tem a região com maior número de casos, possui também duas outras regiões com apenas 23 registros

\section{DISCUSSÃO}

Sabe-se que, no Brasil, a expansão do processo de urbanização - principalmente das áreas periféricas e transicionais dos grandes centros - provocou um crescimento das transformações ambientais antropogênicas e um acelerado êxodo rural, com diversos déficits em infraestrutura e condições sanitárias, propiciando o deslocamento e adequação tanto do vetor da Leishmaniose visceral quanto do seu reservatório ${ }^{5}$. A doença, que antes era restrita a determinadas regiões, adquiriu uma distribuição difusa no país, com rápido aumento no número de casos e certa facilidade em provocar epidemias ${ }^{4}$.

Analisando os dados apresentados no decorrer de 2007 a 2017, pode-se observar que não houve queda ou aumento acentuado dos registros, evidenciando que, desde quando o SINAN iniciou esse apontamento, não houveram melhorias nas políticas de saúde direcionadas a doença - seja quanto ao aumento da detecção de possíveis casos não diagnosticados oportunamente, ou quanto ao controle da infecção por meio de medidas educativas de prevenção. Isso é preocupante, já que a maior exposição ao vetor é um dos principais determinantes para o contágio e corrobora no fato de que a 
maior incidência da doença se dá no sexo masculino durante todos os anos estudados. Esse dado entra em concordância com outros estudos, como o realizado por Souza et al., em Sobral (CE) ${ }^{9}$ nos anos de 2011 a 2015. No caso, não existindo fatores fisiológicos que favoreceriam ao homem apresentar maior número de ocorrências, suspeita-se que isso se dá por conta de uma maior exposição dessa população ao vetor.

As faixas etárias mais acometidas são de 1-4 anos e 20-39 anos, consoante aos demais estudos apreciados: crianças de 1-4 anos são as mais afetadas devido à imaturidade imunológica celular e à presença de subnutrição e desnutrição - agravantes geralmente presentes nas áreas endêmicas, associadas aos baixos níveis socioeconômicos -, além de possuírem menor noção quanto a higiene, prevenção e profilaxia em relação a LV. Além disso, de acordo com Gontijo $^{10}$, em áreas endêmicas do Brasil de LV, $80 \%$ dos acometidos são crianças menores de 10 anos. Os adultos entre 20-39 anos fazem parte do segundo grupo mais afetado devido à uma maior exposição ao vetor, visto que essa faixa etária compreende a população economicamente ativa.

No tocante ao nível de escolaridade, os usuários entre a primeira e a quarta série foram os mais afetados. Contexto semelhante também é percebido em outros estudos de diversas localidades, como o realizado em Palmas (TO) por Jayme et al. ${ }^{11}$, pois, considera-se que a carência de informações quanto a padrões de higiene, prevenção de doenças e educação em saúde aja diretamente na maior taxa de infecção em populações com baixo nível de escolaridade. Ademais, o grande número de casos registrados como "não se aplica" relacionam-se com o fato de que a faixa etária mais acometida (crianças de 1-4 anos) ainda não se encontra na idade escolar.

Sabe-se que a LV pode se desenvolver em pacientes portadores de HIV, sendo considerada uma doença oportunista. Assim, além de a infecção ter um maior potencial de transmissão, recidiva e letalidade devido ao comprometimento do sistema imunológico, a resposta ao tratamento é deficitária em pacientes soropositivos - o que demonstra a importância da busca ativa, registro e acompanhamento longitudinal desses $\operatorname{casos}^{12}$. De acordo com Gontijo $(2004)^{10}$, tem sido crescente o número de indivíduos com co-infecção Leishmania/HIV em áreas urbanas no Brasil, ocorrendo uma nova forma de transmissão através do compartilhamento de agulhas contaminadas.

Quanto à distribuição geográfica, o Nordeste continua sendo a região mais afetada. Esse dado é mais um reflexo da conhecida suscetibilidade das populações socioeconomicamente vulneráveis às doenças negligenciadas, como a LV. Segundo dados do IBGE $(2018)^{13}$, a pobreza aumentou no Brasil, somando-se cerca de 52,5 milhões de pessoas com renda mensal per capita de até 420 reais. Desses, cerca de $47 \%$ residiam no Nordeste e grande parte deles (53\%) viviam no Maranhão - estado que, inclusive, demonstrou a maioria absoluta dos casos de LV no país. Todos os estados da região Nordeste apresentaram indicadores de pobreza acima da média nacional, demonstrando o porquê de esta população também ser mais suscetível à infecção pelo protozoário.

Para mais, segundo a Pesquisa Nacional de Saneamento Básico - PNSB em 2017², dos 5570 municípios, apenas 3206 possuíam serviço de esgotamento sanitário por rede coletora em funcionamento, correspondendo a cerca de $57 \%$ do total. Sabendo-se que as condições de coleta de lixo e saneamento básico influenciam na transmissão da doença, já que o acúmulo de resíduos favorece sua ocorrência, é possível somar às precárias condições de vida da população para reafirmar a necessidade da implantação de novas políticas públicas de saúde ou melhoria das já existentes para diminuir o contágio da doença no país.

Os estados em evidência no número de casos - Maranhão, Minas Gerais, Ceará, Pará, Bahia e Tocantins - apresentaram um padrão ao longo dos anos, não havendo melhoria significativa dos dados, que, muitas vezes, demonstraram piora. Esse contexto pode revelar tanto uma boa capacitação dos profissionais para diagnosticar a doença quanto uma estagnação ou má eficiência dos projetos de controle e erradicação já implantados. No último caso, a integração de serviços de assistência clínica e epidemiológica agregaria valor na implementação de políticas de prevenção e busca ativa e de capacitação profissional bem direcionadas no combate a LV.

Nesse mesmo cenário, tendo conhecimento da população mais vulnerável, mostra-se indispensável o empoderamento social com educação em saúde voltada para a doença, incluindo fatores de risco, transmissibilidade, manifestações clínicas, tratamento e letalidade. Ao munir a comunidade com informações sobre prática preventiva, há maior facilidade de aplicação das políticas implementadas, pois os usuários tornam-se agentes ativos no processo.

\section{CONCLUSÃO}

Diante dos resultados apresentados no presente trabalho, é possível observar que a variação dos casos registrados ao longo dos anos analisados foi pouco heterogênea, não existindo uma diminuição ou aumento consideráveis dos casos. Logo, pode-se inferir que não houve melhoria nas políticas públicas de saúde capazes de diminuir o potencial de transmissão de forma significativa.

Além disso, tem-se que o maior número de casos se dá no sexo masculino, justificado principalmente pela maior exposição dessa população a situações que podem ocasionar a doença, e na faixa etária de 1-4 anos, que, além de possuir uma imaturidade imunológica, apresentam menor instrução e senso crítico de educação em saúde. Pode-se ratificar também que os fatores socioeconômicos possuem relação direta com a transmissibilidade da doença, visto que o Nordeste concentrou o maior número de casos, principalmente o estado do Maranhão - região socioeconomicamente mais vulnerável do país e com altos índices de pobreza.

Considerando a alta incidência e a dificuldade no controle, bem como a relevância do alto número de casos apontados nesse trabalho, mostra-se necessária a implantação de novas políticas de saúde voltadas para a transmissão, busca ativa de casos e reservatórios, bem como a educação em saúde relacionada a LV. Para maior eficácia das medidas de combate e prevenção da doença, deve existir a integração entre os serviços feitos pela vigilância epidemiológica e pela assistência clínica, acesso da população a esses serviços, capacitação dos profissionais de saúde no geral e o empoderamento da população com informações que 
permitam sua contribuição ativa na busca pela redução dos casos.

\section{REFERÊNCIAS}

1- Silva $K B M$ et al. Análise espacial da leishmaniose visceral no município de Palmas, Tocantins, Brasil. Hygeia: Revista Brasileira de Geografia Médica e da Saúde. 2017; v. 13 (25): p. 18. Disponível em <http://www.seer.ufu.br/index.php/hygeia/article/download/36095/ 21001/0 > Acesso em: 15 de agosto de 2019.

2- Leite Al Araújo LB. Leishmaniose visceral: aspectos epidemiológicos relacionados aos óbitos em Mossoró-RN. Revista de Patologia Tropical/Journal of Tropical Pathology. 2013; v. 42 (3). Disponível em: <https://www.revistas.ufg.br/iptsp/article/view/26928> Acesso em: 22 de agosto de 2019.

3- Brasil. Ministério da Saúde. Guia de Vigilância em Saúde. volume único [recurso eletrônico] / Ministério da Saúde, Secretaria de Vigilância em Saúde, Coordenação-Geral de Desenvolvimento da Epidemiologia em Serviços. 3a. ed. Brasília, 2019.

4- Brasil. Ministério da Saúde. Secretaria de Vigilância em Saúde. Departamento de Vigilância Epidemiológica. Manual de vigilância e controle da leishmaniose visceral, 1. a edição, Brasília: Editora do Ministério da Saúde, 2006. Disponível em: <http://bvsms.saude.gov.br/bvs/publicacoes/manual_vigilancia_cont role_leishmaniose_visceral.pdf> Acesso em: 19 de setembro de 2019. 5- Maia-Elkhoury ANS Alves W A Sousa-Gomes ML Sena JM Luna EA. Visceral leishmaniasis in Brazil: trends and challenges. Cad. Saúde Pública [Internet]. 2008. Disponível em: < http://www.scielo.br/scielo.php?script=sci_arttext\&pid=S0102-

$311 \times 2008001200024 \& \operatorname{lng}=e n \& n r m=i s o>$ Acesso em: 23 de setembro de 2019.

6- Alvar J Yactayo S Bern C. Leishmaniasis and poverty. Trends in parasitology; 2006. v. 22 (552-557). Disponível em: <https://www.sciencedirect.com/science/article/pii/S147149220600 239X> Acesso em: 23 de setembro de 2019.

7- Werneck GL Hasselmann MH Gouvea TG. Panorama dos estudos sobre nutrição e doenças negligenciadas no Brasil. Ciênc. saúde coletiva; 2011. v. 16 (39-62). Disponível em: <http://www.scielo.br/scielo.php?script=sci_arttext\&pid=S14138123 2011000100009\&Ing=en\&nrm=iso $>$ Acesso em: 03 de setembro de 2019.

8- IBGE. Censo demográfico, 2019. Disponível em: <www.ibge.gov.br>. Acesso em: 12 out. 2019

9- Sousa NA et al. Perfil epidemiológico dos casos de Leishmaniose visceral em Sobral-CE de 2011 a 2015. SANARE, Revista de Políticas Públicas; 2018. v. 17 (51-57). Disponível em: <https://sanare.emnuvens.com.br/sanare/article/view/1222/653>. Acesso em 20 de agosto de 2020.

10- Gontijo CMF Melo MN Leishmaniose visceral no Brasil: quadro atual, desafios e perspectivas. Rev. bras. Epidemiol; 2004. v. 7 (338349). Disponível em: <http://www.scielo.br/scielo.php?script=sci_arttext\&pid=S1415$790 \times 2004000300011 \& \operatorname{lng}=e n \& n r m=i s o>$. Acesso em 26 de setembro de 2019.

11- Jayme MS Wanderlei CL Moura FFM Castro JGD. Perfil epidemiológico dos casos de Leishmaniose visceral em Palmas, Tocantins no período de 2007-2014. Rev. Pat. Tocantins; 2016. v.3 (61-69). Disponível em: < https://sistemas.uft.edu.br/periodicos/index.php/patologia/article/vi ew/1947/pdf>. Acesso em 20 de agosto de 2020.

12- Brasil. Manual de recomendações para diagnóstico, tratamento e acompanhamento de pacientes com a coinfecção Leishmania-HIV. 1. ed., rev. e ampl. - Brasília: Ministério da Saúde, 2015.

13- IBGE [homepage na internet]. Síntese de Indicadores Sociais. 2018. [acesso em 20 ago 2020]. Disponível em: http:www.ibge.gov.br
14- IBGE, Diretoria de Pesquisas, Coordenação de População e Indicadores Sociais, Pesquisa Nacional de Saneamento Básico 2017. [acesso em 20 ago 2020]. Disponível em: http:www.ibge.gov.br 